\title{
The making of the Litkey Corpus, a richly annotated longitudinal corpus of German texts written by primary school children
}

\author{
Ronja Laarmann-Quante Stefanie Dipper Eva Belke \\ Department of Linguistics \\ Fakultät für Philologie \\ Ruhr-Universität Bochum \\ laarmann-quante|dipper|belkedlinguistics.rub.de
}

\begin{abstract}
To date, corpus and computational linguistic work on written language acquisition has mostly dealt with second language learners who have usually already mastered orthography acquisition in their first language. In this paper, we present the Litkey Corpus, a richly-annotated longitudinal corpus of written texts produced by primary school children in Germany from grades 2 to 4 . The paper focuses on the (semi-)automatic annotation procedure at various linguistic levels, which include POS tags, features of the wordinternal structure (phonemes, syllables, morphemes) and key orthographic features of the target words as well as a categorization of spelling errors. Comprehensive evaluations show that high accuracy was achieved on all levels, making the Litkey Corpus a useful resource for corpus-based research on literacy acquisition of German primary school children and for developing NLP tools for educational purposes. The corpus is freely available under https: / / www. linguistics.rub. de/litkeycorpus/.
\end{abstract}

\section{Introduction $^{1}$}

Language acquisition in modern societies not only concerns learning to understand and produce oral utterances but also how to read and write. Becoming literate in a language is a complex process, and it usually takes years of instruction for learners to master the stylistics of standard written language. At the beginning, learners (of alphabetical languages) first have to learn how to spell the words of their language. This is a non-trivial task because the mapping between spoken sounds and written characters is rarely one-to-one.

Most computational and corpus-based work on written language acquisition has been on L2

\footnotetext{
${ }^{1}$ All URLs provided in this article were checked on May 31st, 2019.
}

data, in particular data from adult learners, e.g. Reznicek et al. (2012). Usually these learners are already literate in their first language so that the concept of mapping sounds to characters, and vice versa, is not new to them, and the focus of research is on identifying (and correcting) grammatical rather than spelling errors (cf., e.g., the shared tasks on grammatical error correction, $\mathrm{Ng}$ et al., 2013, 2014).

Considerably less research has been done on data from children who, for the first time in their life, learn to read and write- - be it in their first language or, for multilingual children, often in their second language. For German, there are some annotated corpora of primary school children's texts: the Osnabrücker Bildergeschichtenkorpus by Thelen (2000, 2010), the Karlsruhe Children's Text Corpus (Berkling et al., 2014; Lavalley et al., 2015), and the H1 and H2 Corpora by Berkling (2016, 2018). All of these corpora provide a target hypothesis for each erroneous spelling, specifying the intended wordform as perceived by the annotator. Except for the Osnabrücker Bildergeschichtenkorpus, the target forms also correct grammatical errors, making it difficult to distinguish between spelling and grammatical competence of the children.

This paper presents the annotation and evaluation of the Litkey Corpus, a longitudinal corpus of written texts in German from children in primary school between grades 2 to 4 . The corpus includes a target hypothesis that corrects for spelling errors only and is richly annotated with linguistic information that relates to spelling and orthography. For example, the word-internal structure (phonemes, syllables and morphemes) and key orthographic features of the target words are provided as well as error tags characterizing the spelling errors in the texts. The paper explains in detail how the corpus was annotated and presents 
an evaluation of the annotation quality. For further information about the composition of the corpus, including rich metadata about the children that provided the texts, see Laarmann-Quante et al. (to appear(b)). The detailed annotation guidelines can be found in Laarmann-Quante et al. (to appear(a)).

The paper is structured as follows. Sec. 2 provides a short introduction to relevant principles of German orthography. Sec. 3 presents the annotation layers, semi-automatic procedures and annotation quality in detail, followed by a conclusion in Sec. 4.

\section{German Orthography}

Following Eisenberg (2006), the basis of German word spelling is formed by correspondences between phonemes and graphemes (PGC mappings) such as $/ 1 / \leftrightarrow<1>^{2}$. These default mappings are frequently overwritten by (i) syllabic, (ii) morphological and/or (iii) morpho-syntactic principles.

(i) For example, the word fallen (['falən], '(to) fall') would be spelled $*<$ falen $>$ according to the default PGC mappings (see Laarmann-Quante et al., to appear(b), for a detailed description of this example). However, one of the syllabic principles requires that the letter that represents a single consonant phoneme between a short stressed and a reduced vowel is doubled, hence the correct spelling is $<$ fallen $>$.

(ii) According to the principle of morpheme constancy, the spelling of a reference form (which is usually a disyllabic word form like fallen) is retained in all other morphologically related word forms. This is why also monosyllabic inflected forms such as <fallt $>$ (['falt], '(you.PL) fall'), $<$ fällt $>$ (['felt], '(he/she/it) falls'), or the derived noun $<$ Fall $>$ (['fal], '(the) fall') are spelled with $<$ ll $>$. Another case of morpheme constancy can be seen in the grapheme $<\ddot{a}>$ in $<$ fällt $>$ : According to the PGC mappings, the $[\varepsilon]$ would be spelled $\langle\mathrm{e}>$, yielding $*<$ fellt $>$. The grapheme $<\ddot{a}>$ contains a visual clue to the morphological relationship between $<$ fällt $>$ and $<$ fallen $>\mid<$ fallt $>\mid<$ Fall $>$ in spite of its different pronunciation.

(iii) Finally, a prominent morpho-syntactic spelling principle is the capitalization of nuclei of

\footnotetext{
${ }^{2}$ Graphemes are marked with $<>$, phonemes with / / and phones with [ ]. Orthographically incorrect spellings are marked with *.
}

\begin{tabular}{ll}
\hline \#Children & 251 (8-11 years; grades 2-4; \\
& $63 \%$ multilingual) \\
\#Elicitations (avg.) & $7.7 \pm 2.1$ texts/child \\
\#Texts & 1,922 \\
\#Tokens / \#Types & 212,505 / 6,364 \\
\hline
\end{tabular}

Table 1: Basic information on the Litkey Corpus

noun phrases. This is why the noun $<$ Fall $>$ '(the) fall' is not spelled $*<$ fall $>$.

\section{Annotations and Annotation Procedures}

The Litkey Corpus is based on a set of texts (manuscripts) collected by Frieg (2014) from 2010-2012. The texts were written by primary school children, who were asked to write down short picture stories, featuring Lea (a girl), Lars (a boy), and Dodo (a dog). Table 1 presents basic statistics on the subset of texts that is used in the Litkey Corpus.

In the context of the Litkey project, the manuscripts were manually transcribed and annotated with a target hypothesis. To assess the quality of these steps, we measured inter-annotator agreement (IAA) among four annotators on a set of ten texts. Across all texts, IAA was high for both the transcription $(95.8 \%$, Fleiss' $\kappa=.98)$ and the target forms $(90.78 \%)$. For more details, see Laarmann-Quante et al. (2017).

Based on the target forms, linguistic and errorrelated information was annotated automatically. This section presents details about the annotations and annotation procedures.

\subsection{POS tagging}

While there are numerous POS taggers for German, it is well known that performance of state-ofthe-art taggers on non-standard data is considerably lower than on standard data, such as newspaper texts (e.g., Giesbrecht and Evert, 2009). Hence we opted for training a specialized POS tagger, which we would then apply to our data, using the STTS tagset (Schiller et al., 1999). A short description of all tags with example words from the Litkey Corpus can be found in Table 7 in the Appendix.

Creating training data As there are no POSannotated corpora of children's text available, we first created training data. To this end, we extracted the grammatical target hypotheses of 
the Osnabrücker Bildergeschichtenkorpus (Thelen, 2000, 2010) and H1 Corpus (Berkling, 2016) (see Sec. 1). These corpora are rather similar to our corpus. For instance, they also include grammatically ill-formed texts without proper sentence boundary marking.

We enriched the texts semi-automatically with POS tags as follows: The data was first tagged independently by two taggers, the TreeTagger (Schmid, 1995) using the standard German model and the Stanford POS Tagger (Toutanova et al., 2003) using the 'hgc' model. For words on which the taggers did not agree, the final tag was chosen manually or semi-automatically by identifying areas in which one of the taggers consistently produced better results. For instance, the TreeTagger performed better than the Stanford Tagger in distinguishing between articles and pronouns (in particular PDS, PIS-i.e., demonstrative and indefinite pronouns).

We manually evaluated a random sample of $10 \%$ of the texts from the Osnabrücker Bildergeschichtenkorpus and $7 \%$ from the $\mathrm{H} 1$ Corpus (one text per class per test date), which showed an overall POS error rate of $2.5 \%$ after processing as described above. ${ }^{3}$

To further improve the quality of the training data, we reviewed unusual tag sequences, such as determiner-determiner, and corrected them manually. A second evaluation on another random sample of the same size, which did not include any of the texts from the previous sample, showed a considerable decrease of the error rate to $1.2 \%$, so approximately one tag in a hundred in the training data is expected to be incorrect.

Training We next trained the Stanford POS Tagger on the training data, using its bidirectional architecture. That is, the tagger considers the previous and the following word as well as one or two previous and following tags to determine the correct tag for a given word. The tagger model was trained to be case-sensitive. This implies that it can take advantage of letter case information, for instance when tagging nouns and proper nouns, which are capitalized in German. This tagger was used to automatically tag the entire Litkey Corpus

\footnotetext{
${ }^{3}$ The most frequent errors were confusions of noun vs. proper name, finite verb vs. infinitive, adverbial or predicative adjective vs. adverb, and coordinating conjunction vs. adverb. Also, no relative pronoun was detected correctly due to missing commas in the children's texts (commas are usually strong indicators of such pronouns in German).
}

without any manual correction.

Test set The test set-which we use for evaluating all automatic annotations (POS, graphemes, morphemes, etc.)—consists of 20 texts chosen randomly from our corpus. The sample amounts to 1,795 target tokens (477 types). Among these, 1,623 target tokens contain at least one alphabetical character (458 types). Average length of target tokens with at least one alphabetical character is $4.4 \pm 1.9$ characters.

Evaluation The gold standard was constructed by one human annotator who tagged all of the tokens manually. Difficult or unclear cases, which constituted less than $1 \%$ of the data, were discussed with two other project members.

The tagger achieved an overall accuracy of $92.81 \%$. This is below state-of-the-art results for standard German, which range from 95-98\% (Giesbrecht and Evert, 2009). However, applying standard taggers to nonstandard web data results in accuracies in the range of 90-94\%, and our tagger's performance is within this range. Given that we trained our model on nonstandard data, one could have expected a better outcome; however, it has to be taken into account that our training base was rather small $(<110,000$ tokens, which corresponds to approximately $10 \%$ of the TIGER Corpus used by Giesbrecht and Evert, 2009). ${ }^{4}$

POS categories which turned out difficult for the tagger include PTKVZ (verb particles, 35\% recall), ITJ (interjections, 61\%), VVINF (infinitives, $67 \%$ ), PAV (pronominal adverbs, $80 \%$ ), XY (nonwords, $80 \%$ ). PTKVZ marks separated verb particles and is notorious for being confounded with adverbs. In addition, our data shows that PTKVZ is confounded with ADJD (adjectives) and APPR (prepositions), probably because many of our texts do not have reliable markers of sentence boundaries. In the Litkey Corpus, XY-words include syntactically unclear cases, like in (1): um could be a separated verb particle but cannot cooccur with runtergefallen, so the gold standard $(\mathrm{G})$ tags it as $\mathrm{XY}$, whereas the tagger (system, S) decided for KOUI.

\footnotetext{
${ }^{4} \mathrm{An}$ idea for future work could be to merge the TIGER Corpus with our nonstandard learner data for training. This kind of procedure has succesfully been applied to texts from computer-mediated communication, see Horbach et al. (2014). Also, the impact of sentence boundary detection would be an interesting further point of study. We thank the reviewers for these suggestions.
} 


\begin{tabular}{|c|c|}
\hline Analysis & Description \\
\hline fröhlich & Original input \\
\hline fr'2:.1IC & $\begin{array}{l}\text { Phonemes with stress marks (') } \\
\text { and syllable boundaries (.) in } \\
\text { SAMPA notation (Wells, 1997) }\end{array}$ \\
\hline fröh lich & Morphemes (space-separated) \\
\hline ADJ SFX & $\begin{array}{l}\text { Morpheme tags (adjective stem } \\
\text { and suffix) }\end{array}$ \\
\hline
\end{tabular}

Table 2: BAS' G2P analysis for fröhlich 'happy' $\begin{array}{llll}\text { Fast hat der } & \text { Turm um runtergefallen } \\ \text { almost has } & \text { the tower? } & \text { down fallen }\end{array}$ S: ADV VAFIN ART NN KOUI XY

G: ADV VAFIN ART NN XY VVPP 'The tower has almost fallen down'

\subsection{Word-internal structure}

For each target word (type), we obtained information on the word-internal structure from the web service G2P of the Bavarian Archive of Speech Signals (BAS) (Reichel, 2012; Reichel and Kisler, 2014). ${ }^{5}$ Table 2 shows the (reformatted) output of the G2P web service for the word fröhlich 'happy'. ${ }^{6}$

The following paragraphs explain how we processed G2P's output in the Litkey Corpus. For evaluating these word-internal analyses, the test set of 1,623 tokens with at least one alphabetical character was used (458 types).

\subsubsection{Phonemes and PCUs}

We aligned the characters of our target forms with G2P phonemes, to form phoneme-corresponding units (PCUs). ${ }^{7}$ How this was achieved automatically is described in detail in Laarmann-Quante

\footnotetext{
${ }^{5}$ https: / / clarin.phonetik. uni-muenchen. de/BASWebServices/interface/ Grapheme2Phoneme.

The following parameters were set: "lng":"deu-DE", "syl":"yes", "stress":"yes", "iform": "list", "oform":"exttab", "featset":"extended".

${ }^{6}$ The original G2P output also provides POS tags. However, for efficiency reasons, we used the G2P web service to analyze individual words (types) rather than word sequences. As a result, the web service's analysis of POS tags was not informed by a word's phrasal or sentential context, which is why we expected our own tagger to outperform the webservice's tagger and decided to ignore G2P's POS tags.

Similarly, G2P provides an alignment of phonemes and graphemes. However, the tool often had problems aligning words with $\langle\mathrm{x}>/[\mathrm{ks}]$ or $\langle\mathrm{z}>/[\mathrm{ts}]$, so we did not use it.

${ }^{7} \mathrm{G} 2 \mathrm{P}$ provides a phoneme analysis for all words but we decided to exclude some types of words like abbreviations from receiving a phoneme annotation in the Litkey Corpus. For details, see Laarmann-Quante et al. (to appear(b)).
}

(2016). In summary, we first statistically determined a $1: 1$ (or 1:0, 0:1) mapping of phonemes and characters based on cost-weighted Levenshtein distance ${ }^{8}$, see (2).

(2)

\begin{tabular}{|l|c|c|c|c|c|c|c|c|}
\hline Characters & f & r & ö & h & l & i & c & h \\
\hline Phonemes & f & r & 2 & $:$ & l & I & & C \\
\hline
\end{tabular}

Next, we applied hand-coded rules to merge those characters which together correspond to one phoneme, and those phonemes which together correspond to one grapheme. An example is given in (3); here, merged PCUs are $<$ öh $>\approx / 2: /$ and $<\mathrm{ch}>\approx / \mathrm{C} /$.

(3)

\begin{tabular}{|l|c|c|c|c|c|c|}
\hline Characters & f & r & öh & l & i & ch \\
\hline Phonemes & f & r & $2:$ & l & I & C \\
\hline
\end{tabular}

We evaluated the accuracy of the PCUs on our test set. Two independent raters, who reconciled cases of disagreement in subsequent discussions, judged for each PCU whether the PCU was correctly aligned ("c") or false ("f"). Cases where the G2P phoneme was incorrect were also marked as false ("f"). We also marked missing ("m"), or superfluous ("s") phonemes. When in doubt about a pronunciation, the Duden pronunciation dictionary (Mangold, 2005) was used as a reference. IAA was $97.7 \%$, Cohen's $\kappa=.70 .^{9}$ Example (4a) provides cases of incorrect alignments in the word Angst 'fear', (4b) shows a missing phoneme and an incorrect G2P phoneme in the analysis of the proper name Lars.

(4) $\mathrm{a}$

\begin{tabular}{|l|c|c|c|c|c|c|}
\hline Chars & & A & n & g & s & t \\
\hline G2P & & ? & a & N & s & t \\
\hline Gold & ? & a & \multicolumn{2}{|c|}{ N } & s & t \\
\hline \hline Raters & m & f & f & f & c & c \\
\hline
\end{tabular}

b.

\begin{tabular}{|l|c|c|c|c|}
\hline Chars & L & a & r & s \\
\hline G2P & l & a & & S \\
\hline Gold & l & a & r & s \\
\hline \hline Raters & c & c & m & f \\
\hline
\end{tabular}

Table 3 displays the result of the PCU/phoneme evaluation (see second column): $96.19 \%$ of the PCUs are correct, i.e., the aligned G2P and gold

\footnotetext{
${ }^{8}$ Using a script created by Marcel Bollmann, https : / / github.com/mbollmann/levenshtein/.

${ }^{9}$ The $\mathrm{R}$ package irr was used for computing agreement, https : / / CRAN.R-project.org/package=irr.
} 
phonemes are identical. At the word level, 90.33\% of the tokens and $94.04 \%$ of the types receive a completely correct PCU/phoneme analysis. ${ }^{10}$

We went through all incorrect cases again and decided which errors are due to incorrect alignments (all cases of "f" in (4a)) and which ones are due to incorrect G2P phonemes ("f" in (4b) and all cases of "m" and "s"). ${ }^{11}$ It turned out that incorrect alignments ("false boundary") are only a minor problem. Similarly, missing or superfluous units play virtually no role.

After the evaluation, we decided to further improve the quality of the phoneme annotations in our corpus by manually correcting the G2P phoneme analyses for all target types in the entire corpus. ${ }^{12}$ In total, 1,184 of 6,340 types underwent a correction in that step.

\subsubsection{Graphemes}

We identified multi-letter graphemes automatically based on PCUs as follows: Whenever one of the sequences $<\mathrm{ie}>,<\mathrm{qu}>,<\mathrm{ch}>$, or $<\mathrm{sch}>$ was found within a PCU, we considered it a single grapheme, as in Flasche 'bottle', see (5a). Otherwise we split it into several graphemes, as in bisschen 'a little', see (5b). The evaluation showed that grapheme identification was almost perfect: in just two cases, a grapheme was analyzed incorrectly. ${ }^{13}$

(5) $\mathrm{a}$

\begin{tabular}{|l|l|l|l|l|l|}
\hline Graphemes & F & 1 & a & sch & e \\
\hline Phonemes & f & 1 & a & S & $@$ \\
\hline
\end{tabular}

b.

\begin{tabular}{|l|l|l|l|l|l|l|}
\hline Graphemes & b & i & ss & ch & e & n \\
\hline Phonemes & b & I & s & C & $@$ & n \\
\hline
\end{tabular}

\subsubsection{Syllables}

For each word (type), the G2P web service marks the syllable boundaries and assigns exactly one stressed syllable (see Table 2). G2P records these

\footnotetext{
${ }^{10}$ The difference between the token and type level can be explained by the fact that some high-frequency words in the corpus were analyzed incorrectly, such as Lars, see (4b).

${ }^{11}$ Some cases of " $\mathrm{m}$ " and "s" could alternatively be analyzed as follow-up errors of an incorrect alignment, as in (4a).

${ }^{12}$ Some rare cases of homographs with differing pronunciations would have required knowledge of the actual context, which we did not have in the correction step since we considered types instead of tokens. In such cases, the most common usage was chosen for the annotation. An example is so, which can be read (in IPA) as [zo:] ('this way') or [zo] (interjection similar in meaning to 'right!') and was annotated as [zo:].

${ }^{13}$ This was due to a bug in the script, which has been fixed.
}

features at the phoneme level. In the Litkey Corpus, we moved these features to the level of the target characters so that we are able to make statements about a character's position in a syllable. This is particularly relevant for ambisyllabic consonants: In syllable joints, an ambisyllabic phoneme belongs to the coda of the first and the onset of the second syllable at the same time, e.g., /t/ in Ratte ([rat@], 'rat'). At the grapheme level, an ambisyllabic phoneme usually corresponds to a doubled consonant (e.g., $<\mathrm{tt}>$ ) or another consonant pair (such as $\langle\mathrm{ck}\rangle,\langle\mathrm{tz}\rangle$, or $\langle\mathrm{ng}\rangle$ ). In these cases, the orthographic syllable boundary is placed between these consonants $(<$ Rat.te $>$ 'rat', <Jac.ke> 'jacket'). ${ }^{14}$

The G2P phoneme representation only distinguishes between (one) stressed syllable vs. unstressed syllables in a word. We introduced a third category, reduced, using the following heuristics: each syllable with a G2P stress mark is classified as stressed, each syllable that has [@] or [6] as its nucleus is a reduced syllable, and the rest is classified as unstressed.

We evaluated syllable boundaries and syllable types (stressed, unstressed, reduced) in the same way as PCUs (see above). IAA was $97.3 \%$, Cohen's $\kappa=.79$. Overall system accuracy is $91.84 \%$ (see Table 3, third column), and word-level accuracy is $93.04 \%$ (tokens) and $87.16 \%$ (types). ${ }^{15}$ Compared to PCUs/phonemes, labeling was easier for syllables as there are only three types to choose between. Incorrect boundaries, which make up two thirds of the errors, are either wrong in the G2P output from the start or the G2P boundaries had been correct initially but were spoilt by mapping them from the phoneme to the character level.

As in the case of phonemes, we made some efforts after the evaluation to further improve the annotations. We made minor adjustments to the syllable scripts and manually corrected all syllable boundary and stress marks in the G2P output for all target types in our corpus.

\footnotetext{
${ }^{14}$ An exception are the multi-letter graphemes $<\mathrm{ch}>$ and $<$ sch >: they can correspond either to a syllable-initial phoneme, as in Suche ([zu:.x@] 'search', or to an ambisyllabic phoneme, as in Sache ([zax@] 'thing'). Here, we placed the boundaries always in front of the respective grapheme: $<$ Su.che $>,<$ Sa.che $>$.

${ }^{15}$ In $96.24 \%$ of the word tokens ( $94.72 \%$ of types), at least one syllable was analyzed correctly.
} 


\begin{tabular}{lrrr}
\hline Linguistic Unit & PCUs/Phonemes & Syllables & Morphemes \\
\hline Total number & 6,690 & 2,378 & 2,278 \\
Correct & $96.19 \%$ & $91.84 \%$ & $82.88 \%$ \\
False & $2.44 \%$ & $8.07 \%$ & $13.56 \%$ \\
$\quad$ among them: false boundary ${ }^{a}$ & $6.13 \%$ & $67.19 \%$ & $25.89 \%$ \\
$\quad$ among them: false label ${ }^{a}$ & $95.09 \%$ & $34.38 \%$ & $82.52 \%$ \\
Missing & $1.38 \%$ & $0.08 \%$ & $3.56 \%$ \\
Superfluous $^{b}$ & $<0.01 \%$ & $<0.01 \%$ & $<0.01 \%$ \\
Correct word tokens $(1623)^{c}$ & $90.33 \%$ & $93.04 \%$ & $85.21 \%$ \\
Correct word types (436) $^{c, d}$ & $94.04 \%$ & $87.16 \%$ & - \\
\hline
\end{tabular}

Table 3: Evaluation of the analysis of a word's internal structure based on the BAS web service G2P

${ }^{a}$ The figures for false boundary and false label do not add up to $100 \%$ because both the boundary and the label can be wrong at the same time.

${ }^{b}$ The proportion of superfluous elements was calculated as $\frac{\# \text { \#superfluous }}{\text { \#gold-phonemes }}$. Note that there could be more than $100 \%$ superfluous elements, and there is no upper bound.

${ }^{c}$ Letter case is usually irrelevant for phoneme and syllable annotation, so word types are case-insensitive here.

${ }^{d}$ Since certain morpheme categories are context-dependent, they cannot be evaluated on word types but only on word tokens.

\subsubsection{Morphemes}

Morphemes can be either stems or affixes, and are tagged accordingly (see Table 2). While suffix morphemes are always unambiguous (just like phonemes, PCUs, and syllables), certain stem morphemes can only be determined in the phrasal or sentential context. For example, the stem $d$ may be an article (ART) or a demonstrative pronoun (PD) depending on the context, see (6). In the examples, morphemes are separated by hyphens, and corresponding glosses and morpheme tags are marked in the same way.

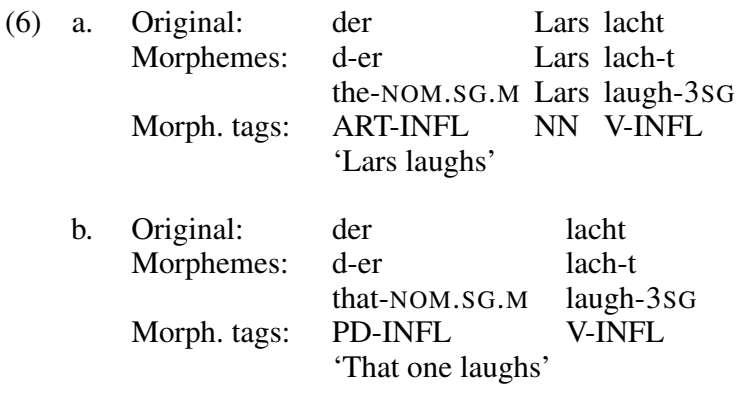

For efficiency reasons, we used G2P to analyze the morphemes of word types, i.e., G2P's analyses were not informed by a word's phrase or sentence contexts (also see Footnote 6). To integrate this information in the annotations, we fed the analysis of our POS tagger into the morpheme analysis: whenever a word consisted of one stem morpheme only, or one stem morpheme followed by an INFLmorpheme, the word's POS tag was used to derive the tag for the stem morpheme.
This fixed certain errors introduced by G2P. For instance, for a verb whose stem coincides with an existing noun stem, G2P often analyzed the stem as a noun, as in (7): the verb stem wein- is also a noun stem, Wein ('wine'). Looking at the POS tag, VVFIN, it becomes clear that it is the verb stem in this case.

(7)

$\begin{array}{ll}\text { Original: } & \text { weint 'cries' } \\ \text { Morphemes: } & \begin{array}{l}\text { wein-t } \\ \text { cry-3SG }\end{array} \\ \text { G2P analysis: } & \text { N-INFL } \\ \text { corrected: } & \text { V-INFL } \\ \text { POS tag: } & \text { VVFIN }\end{array}$

For words with two morphemes one of which has the type INFL, we found that replacing the G2P stem morpheme tag based on the POS information of the full word form yielded an overall improvement in accuracy of 2.9 percentage points for morphemes and 3.7 percentage points for tokens. However, some instances were negatively affected by this procedure, e.g. verb stems that are derived from a noun via conversion, such as teil-t 'shares', which is derived from Teil 'part'.

We evaluated the automatic morpheme analysis on the test set in the same way as the PCUs presented above. The raters used the online grammar canoonet ${ }^{16}$ as a reference when they were in doubt about a word's morphological structure. IAA was $89.9 \%$, Cohen's $\kappa=.66$.

\footnotetext{
${ }^{16}$ http: / / canoo. net/.
} 
Table 3 (fourth column) shows that $82.88 \%$ of the morphemes and $85.21 \%$ of the tokens are analyzed correctly by the system (in $90.02 \%$ of the tokens at least one morpheme has been identified correctly in terms of label and boundaries). Similarly to PCUs, selecting the label was more errorprone than establishing the morpheme boundaries.

The most problematic tags, which have a recall below 75\%, are ITJ (interjections, 47.6\%), SFX (suffixes, 50.0\%), PRFX (prefixes, 64.5\%), and INFL (inflection, 74.7\%). It is noteworthy, however, that confusions of tags are mainly found within categories for stems (e.g., nouns vs. verbs) or affixes (e.g., INFL vs. SFX) rather than across categories.

This time, we did not correct the morpheme analyses manually after the evaluation, in contrast to phonemes and syllables, because some morphemes are context-dependent and a correction would have required that we assess each morpheme in context.

\subsection{Key orthographic features}

The focus of the Litkey project is on analyzing orthographic errors. To this end, we developed a scheme of fine-grained spelling categories (see Laarmann-Quante et al., to appear(a), for a detailed presentation). These categories are annotated at the PCUs and specify detailed orthographic properties of the respective $\mathrm{PCU}$ in its context. For instance, the PCU <öh> $\approx / 2: /$ in (3) is annotated with the spelling category Vlong_single_h, which specifies that the letter $<\mathrm{h}>$ marks a (preceding) single vowel as long. The spelling categories are purely descriptive and are intended to highlight locations where errors are likely to occur.

On top of the highly specific spelling categories, we define more general key orthographic features (KOFs), which encode important spelling-related properties of the word (see Sec. 2) and are inspired by categories as they are used in teaching contexts. Table 6 in the Appendix provides a list of all KOFs (for more details, see Laarmann-Quante et al., to appear(b)).

Technically, all KOFs are derived from the finegrained spelling categories. Some KOFs match some spelling categories exactly. For example, if final devoicing is a spelling category on a given word (category final_devoice), this word is assigned the KOF devoice_final. In some cases, however, KOFs are not purely descriptive (in contrast to the fine-grained spelling categories) but relate the PCUs to the spelling principles. For instance, the spelling categories for doubled consonants within a morpheme only describe the context, e.g., Cdouble_interV specifies that the doubled consonants occur between vowels; Cdouble_beforeC means that it occurs before another consonant.

The corresponding KOFs, in contrast, distinguish between those doubled consonants that arise from a syllabic principle (see Sec. 2) and those which do not. For instance, alle (['al@], 'all') is an example of consonant doubling due to syllabic constraints (KOF: doubleC_syl), namely because there is a single consonant letter between a short stressed and an unstressed vowel. In allein ([a'laIn], 'alone'), the doubled consonant is between an unstressed and a stressed vowel, which is a marked stress pattern. Here, the doubling cannot be explained synchronically (hence, KOF: doubleC_other). So in order to determine automatically which kind of consonant doubling is present, information about a word's syllable and morpheme structure is necessary.

We evaluated the automatic analysis of KOFs based on 427 types from our test set (excluding words marked as ungrammatical or unidentifiable). Five independent raters judged for each word and each KOF whether the word features this KOF, possibly more than once. For example, the word Staubsauger ([StaUpsaUg6], 'vacuum cleaner') contains three instances of the KOF graph_comb $(<\mathrm{St}>,<\mathrm{au}>,<\mathrm{au}>)$, and one instance each of devoice_final $(<b>)$ and r_voc $(<e r>)$. Together the raters agreed on a gold standard, using the pronunciation Duden (Mangold, 2005) as a reference.

The evaluation results in Table 4 specify correct ("c"), missing ("m") and superfluous ("s") KOFs and provide precision and recall scores for each KOF. While most features were determined automatically with high accuracy, the detection of doubleC_other was problematic. Three types of doubleC_other were annotated incorrectly as doubleC_syl (e.g., Uff 'Phew!', Bumm 'Boom!'). This happened mainly because the evaluation was type based, i.e., without context information, causing the tagger to assign incorrect POS tags in some places. This resulted in incorrect morpheme analyses, which are one of the criteria for distinguish- 


\begin{tabular}{lrrrrr}
\hline KOF & $\mathrm{c}$ & $\mathrm{m}$ & $\mathrm{s}$ & Prec & Rec \\
\hline graph_comb & 104 & 1 & 0 & 1.00 & 0.99 \\
graph_marked & 26 & 2 & 0 & 1.00 & 0.93 \\
ie & 28 & 0 & 0 & 1.00 & 1.00 \\
schwa_silent & 40 & 4 & 0 & 1.00 & 0.91 \\
doubleC_syl & 71 & 7 & 3 & 0.96 & 0.91 \\
doubleC_other & 4 & 3 & 6 & 0.40 & 0.57 \\
doubleV & 3 & 0 & 0 & 1.00 & 1.00 \\
h_length & 12 & 0 & 0 & 1.00 & 1.00 \\
h_sep & 10 & 0 & 2 & 0.83 & 1.00 \\
r_voc & 100 & 0 & 7 & 0.93 & 1.00 \\
devoice_final & 72 & 4 & 3 & 0.96 & 0.95 \\
g_spirant & 4 & 0 & 2 & 0.67 & 1.00 \\
morph_bound & 1 & 0 & 0 & 1.00 & 1.00 \\
\hline
\end{tabular}

Table 4: Evaluation results of key orthographic features; "c": correct, "m": missing, "s": superfluous

ing doubleC_syl from doubleC_other. For annotating the corpus, though, the POS tagger can make use of the context, and the KOF annotations of these types are mostly correct. On the other hand, six types were annotated as doubleC_other instead of doubleC_syl due to minor errors in the processing pipeline, which have been fixed in the meantime.

\subsection{KOF errors}

Apart from the key orthographic features that a target word contains, the Litkey Corpus also shows which KOFs are violated in a child's spelling. Take the word annehmen, which contains the two KOFs morph_bound $(<\mathrm{nn}>)$ and $\mathrm{h} \_$length $(<\mathrm{eh}>)$. If the word was misspelled as *<anehmen $>$, the error would violate the KOF morph_bound; *<annemen>, by contrast, would pertain to KOF h_length. Any other error, e.g., *<Annehmen $>$, would not affect a KOF.

Like the KOFs, KOF errors are derived from the more fine-grained spelling categories. We evaluated the automatic annotation of KOF errors on 317 types from our test set. A type consisted of a pair of original and target spelling. Three human annotators established the gold standard in that they determined the KOF error categories that applied to a misspelling. The position of the error in a word was not annotated. 115 words contained more than one error, resulting in 475 errors in total. An example annotation is given in (8). The KOF error category "other" indicates that there was one other error which did not pertain to a KOF (in this case, the incorrect capitalization).

\begin{tabular}{lr}
\hline KOF error & count \\
\hline other & 293 \\
doubleC_syl & 63 \\
hyp & 29 \\
ie & 18 \\
graph_marked & 17 \\
r_voc & 12 \\
devoice_final & 9 \\
h_sep & 8 \\
h_length & 8 \\
doubleC_other & 7 \\
graph_comb & 5 \\
doubleV & 3 \\
g_spirant & 2 \\
morph_bound & 1 \\
\hline
\end{tabular}

Table 5: KOF errors occurring in the test set (based on the gold standard)

$\begin{array}{lll}\text { (8) } & \text { orig } & \text { Felt } \\ \text { target } & \text { fällt } \\ \text { KOF errors } & \text { doubleC_syl,graph_marked,other }\end{array}$

Table 5 shows the distribution of KOF error categories in the test set. The majority of errors falls under "other", which subsumes all errors not pertaining to a KOF. The KOFs were chosen to reflect instances of syllabic spelling principles and morpheme constancy, where the correct spelling deviates from default phoneme-grapheme mappings. The category "other" includes some highly frequent errors pertaining to morpho-syntax such as capitalization as well as violations of regular phoneme-grapheme mappings (e.g. $*<$ brcht $>$ for $*<$ bricht $>$ '(it) breaks').

For the evaluation, the automatically generated set of KOF errors for a word was compared to the manually created one. When the two did not match completely, the automatic annotation was considered incorrect. Since in this evaluation we did not mark the position of individual errors, the system categories could not be mapped onto the gold categories. Hence, an analysis of which categories were missed or confused by the automatic script was not possible. In total, 281 (88.6\%) origtarget pairs were analyzed correctly and 36 incorrectly. Of these, 23 contained words with more than one KOF error in the gold standard, which shows that these pose a particular challenge to the automatic analysis. 


\section{Conclusion and Outlook}

This paper presents annotations and annotation procedures for the Litkey Corpus, a longitudinal corpus of written texts produced by German primary school children. Besides categorization of spelling errors, the annotations include information on POS, the word-internal structure (phonemes, syllables, morphemes), and key orthographic features of the target words. Evaluations of all annotations show high accuracy, so that we believe that the corpus can serve as a reliable resource for research on literacy acquisition and for the development of NLP tools in educational contexts. Using the corpus, research questions that have so far only been addressed using experimental methods (i.e., with small, pre-selected sets of materials), can now be addressed on a larger scale and based on spellings that were produced spontaneously rather than spellings that were produced on dictation. In addition, the corpus allows for longitudinal studies of spelling acquisition, which is particularly helpful for studies on the role of implicit learning in spelling acquisition. Here, the question is to what extent cues that are not taught at school can influence the acquisition of word spellings. Such cues are likely to be of a statistical nature, such as bigram frequencies or syllable frequencies or orthographic consistency. Experimental studies (e.g., de Bree et al., 2018; Treiman and Wolter, 2018) suggest that implicit cues have a substantial impact on the acquisition of vowel spellings and double consonant spellings.

The Litkey Corpus is available via the website https://www. linguistics.rub.de/

litkeycorpus/ under the Creative Commons Attribution-ShareAlike 4.0 license (CC BY-SA 4.0). It comes in different formats, including a custom-made XML format (see Laarmann-Quante et al., 2016) and a tabular format including information on types and tokens, respectively, and their annotations (see Laarmann-Quante et al., to appear(b)). The corpus can also be searched via the corpus search tool ANNIS (Krause and Zeldes, 2016). For future work, we plan to enrich the corpus with annotations on grammatical errors as well.

\section{Acknowledgments}

This research is part of the project Literacy as the key to social participation: Psycholinguistic perspectives on orthography instruction and literacy acquisition funded by the Volkswagen Foundation as part of the research initiative "Key Issues for Research and Society" (grant no. I/89 479). We would also like to thank the anonymous reviewers for their helpful comments.

\section{References}

Kay Berkling. 2016. Corpus for children's writing with enhanced output for specific spelling patterns (2nd and 3rd grade). In Proceedings of the Tenth International Conference on Language Resources and Evaluation (LREC 2016), pages 3200-3206.

Kay Berkling. 2018. A 2nd longitudinal corpus for children's writing with enhanced output for specific spelling patterns. In Proceedings of the Eleventh International Conference on Language Resources and Evaluation (LREC 2018), pages 2262-2268.

Kay Berkling, Johanna Fay, Masood Ghayoomi, Katrin Heinz, Rémi Lavalley, Ludwig Linhuber, and Sebastian Stüker. 2014. A database of freely written texts of German school students for the purpose of automatic spelling error classification. In Proceedings of the Ninth International Conference on Language Resources and Evaluation (LREC 2014), pages 12121217.

Elise de Bree, Jan Geelhoed, and Madelon van den Boer. 2018. Overruled! Implicit cues rather than an orthographic rule determine Dutch children's vowel spelling. Learning and Instruction, 56:30-41.

Peter Eisenberg. 2006. Das Wort, 3rd edition, volume 1 of Grundriss der deutschen Grammatik. J.B. Metzler, Stuttgart.

Hendrike Frieg. 2014. Sprachförderung im Regelunterricht der Grundschule: Eine Evaluation der Generativen Textproduktion. Ph.D. thesis, Ruhr-Universität Bochum.

Eugenie Giesbrecht and Stefan Evert. 2009. Is part-ofspeech tagging a solved task? An evaluation of POS taggers for the German web as corpus. In Proceedings of the fifth Web as Corpus workshop (WAC5), pages $27-35$.

Andrea Horbach, Diana Steffen, Stefan Thater, and Manfred Pinkal. 2014. Improving the performance of standard part-of-speech taggers for computermediated communication. In KONVENS 2014, pages $171-177$.

Thomas Krause and Amir Zeldes. 2016. ANNIS3: A new architecture for generic corpus query and visualization. Digital Scholarship in the Humanities, 31(1):118-139.

Ronja Laarmann-Quante. 2016. Automating multilevel annotations of orthographic properties of German words and children's spelling errors. In Proceedings of the 2nd Language Teaching, Learning and Technology Workshop (LTLT), pages 14-22. 
Ronja Laarmann-Quante, Anna Ehlert, Katrin Ortmann, Doreen Scholz, Carina Betken, Lukas Knichel, Simon Masloch, and Stefanie Dipper. to appear(a). The Litkey spelling error annotation scheme: Guidelines for the annotation of orthographic errors in German texts. Bochumer Linguistische Arbeitsberichte (BLA).

Ronja Laarmann-Quante, Lukas Knichel, Stefanie Dipper, and Carina Betken. 2016. Annotating spelling errors in German texts produced by primary school children. In Proceedings of the 10th Linguistic Annotation Workshop held in conjunction with ACL 2016 ( $L A W-X 2016$ ), pages 32-42.

Ronja Laarmann-Quante, Katrin Ortmann, Anna Ehlert, Simon Masloch, Doreen Scholz, Eva Belke, and Stefanie Dipper. to appear(b). The Litkey Corpus: A richly annotated longitudinal corpus of German texts written by primary school children. Behavior Research Methods.

Ronja Laarmann-Quante, Katrin Ortmann, Anna Ehlert, Maurice Vogel, and Stefanie Dipper. 2017. Annotating orthographic target hypotheses in a German L1 learner corpus. In Proceedings of the 12th Workshop on Innovative Use of NLP for Building Educational Applications (BEA), pages 444-456.

Rémi Lavalley, Kay Berkling, and Sebastian Stüker. 2015. Preparing children's writing database for automated processing. In Workshop on L1 Teaching, Learning and Technology (LITLT), pages 9-15.

Max Mangold. 2005. Duden (Band 6). Das Aussprachewörterbuch, 6th edition. Dudenverlag, Mannheim.

Hwee Tou Ng, Siew Mei Wu, Ted Briscoe, Christian Hadiwinoto, Raymond Hendy Susanto, and Christopher Bryant. 2014. The CoNLL-2014 shared task on grammatical error correction. In Proceedings of the Eighteenth Conference on Computational Natural Language Learning: Shared Task, pages 1-14.

Hwee Tou Ng, Siew Mei Wu, Yuanbin Wu, Christian Hadiwinoto, and Joel Tetreault. 2013. The CoNLL2013 shared task on grammatical error correction. In Proceedings of the Seventeenth Conference on Computational Natural Language Learning: Shared Task, pages 1-12. Association for Computational Linguistics.

Uwe D. Reichel. 2012. PermA and Balloon: Tools for string alignment and text processing. In INTERSPEECH.

Uwe D. Reichel and Thomas Kisler. 2014. Languageindependent grapheme-phoneme conversion and word stress assignment as a web service. In R. Hoffmann, editor, Elektronische Sprachverarbeitung: Studientexte zur Sprachkommunikation 71, pages 42-49. TUDpress.
Marc Reznicek, Anke Ludeling, Cedric Krummes, Franziska Schwantuschke, Maik Walter, Karin Schmidt, Hagen Hirschmann, and Torsten Andreas. 2012. Das Falko-Handbuch. Korpusaufbau und Annotationen Version 2.01. Berlin, Germany.

Anne Schiller, Simone Teufel, Christine Stöckert, and Christine Thielen. 1999. Guidelines für das Tagging deutscher Textcorpora mit STTS. Universities of Stuttgart and Tübingen.

Helmut Schmid. 1995. Improvements in part-ofspeech tagging with an application to German. In Proceedings of the ACL SIGDAT-Workshop.

Tobias Thelen. 2000. Osnabrücker Bildergeschichtenkorpus: Version 1.0.0.

Tobias Thelen. 2010. Automatische Analyse orthographischer Leistungen von Schreibanfängern. Ph.D. thesis, Universität Osnabrück.

Kristina Toutanova, Dan Klein, Christopher D. Manning, and Yoram Singer. 2003. Feature-rich part-ofspeech tagging with a cyclic dependency network. In Proceedings of the 2003 Conference of the North American Chapter of the Association for Computational Linguistics on Human Language Technology - NAACL '03, pages 173-180. Association for Computational Linguistics.

Rebecca Treiman and Sloane Wolter. 2018. Phonological and graphotactic influences on spellers' decisions about consonant doubling. Memory \& Cognition, 46(4):614-624.

John C. Wells. 1997. SAMPA computer readable phonetic alphabet. In Dafydd Gibbon, Roger Moore, and Richard Winski, editors, Handbook of standards and resources for spoken language systems. Mouton de Gruyter, Berlin, New York. 


\section{Appendix}

\begin{tabular}{|c|c|c|c|}
\hline KOF & Princ & Description & Examples \\
\hline graph_comb & - & $\begin{array}{l}\text { Grapheme combinations: Graphemes for phoneme combi- } \\
\text { nations that could be spelled by combining the individual } \\
\text { graphemes but that have an idiosyncratic spelling (e.g., <qu> } \\
\text { for }[\mathrm{kv}],<\mathrm{eu}>\text { for }[\mathrm{OY}]) \text {. }\end{array}$ & $\begin{array}{l}<\mathrm{sp}>,<\mathrm{st}>,<\mathrm{ei}>,<\mathrm{ai}>,<\mathrm{eu}> \\
<\mathrm{äu}>,<\mathrm{au}>,<\mathrm{qu}>\end{array}$ \\
\hline graph_marked & PG & $\begin{array}{l}\text { Marked graphemes: Graphemes for which other graphemes } \\
\text { would be available by default (e.g., <ai }>\text { is a marked } \\
\text { grapheme for [aI], which is spelled <ei>, by default). }\end{array}$ & $\begin{array}{l}<\mathrm{ai}>,<\mathrm{äu}>,<\mathrm{a}>,<\mathrm{y}>,<\mathrm{c}> \\
(\text { except in }<\mathrm{ch}>,<\mathrm{sch}>,<\mathrm{ck}>), \\
<\mathrm{chs}>,<\mathrm{ks}>,<\mathrm{dt}>,<\mathrm{th}>,<\mathrm{v}>, \\
<\mathrm{ph}>,<\mathrm{ts}>\end{array}$ \\
\hline ie & PG & $\begin{array}{l}<i e>\text { : A special grapheme in that it is the only multi-letter } \\
\text { grapheme for a tense vowel /i/; the lax counterpart, /I/, is } \\
\text { mapped onto }<\mathrm{i}>\text {. All other pairs of tense and lax vow- } \\
\text { els (e.g., /y/-/Y/) are mapped onto the same single-letter } \\
\text { grapheme by default. }\end{array}$ & $<\mathrm{ie}>$ \\
\hline schwa_silent & SL & $\begin{array}{l}\text { Silent schwa: In reduced syllables [@] is often not audible in } \\
\text { words ending with /@m/, /@n/, and /@1/. Irrespective of this, } \\
\text { the spelling of all reduced syllables including a silent schwa } \\
\text { always includes an }<\mathrm{e}>\text {. }\end{array}$ & Hasen 'rabbits' \\
\hline doubleC_syl & SL & $\begin{array}{l}\text { Double consonant spellings: In disyllabic words with the de- } \\
\text { fault German stress pattern (trochee: stressed-unstressed, typ- } \\
\text { ically stressed-reduced), doubled consonants indicate to the } \\
\text { reader the laxness/shortness of the first vowel; doubleC_syl is } \\
\text { also annotated in word forms for which morpheme constancy } \\
\text { requires that the double consonant spelling is carried forth } \\
\text { from a reference form. }\end{array}$ & fallen/fällt '(to) fall/(s/he) falls' \\
\hline doubleC_other & - & $\begin{array}{l}\text { Other double consonants: Consonant doublings which can } \\
\text { neither be explained via the word's syllabic structure, nor } \\
\text { morpheme constancy, nor a morpheme boundary. }\end{array}$ & dann 'then', jetzt 'now' \\
\hline doubleV & SL & $\begin{array}{l}\text { Double vowels: Indicate the length of tense vowels in stressed } \\
\text { syllables. }\end{array}$ & Seelen 'souls' \\
\hline h_length & SL & $\begin{array}{l}\text { Vowel-lengthening }<\mathrm{h}>\text { : Indicates the length of tense vowels } \\
\text { in stressed syllables. }\end{array}$ & Kehlen 'throats' \\
\hline h_sep & SL & $\begin{array}{l}\text { Syllable-separating }<\mathrm{h}>\text { : Indicates separate syllables in the } \\
\text { spelling of words that include two adjacent vowels belonging } \\
\text { to different syllables; } \mathrm{h} \text { _sep is also annotated in word forms } \\
\text { for which morpheme constancy requires that the syllable- } \\
\text { separating }<\mathrm{h}>\text { is carried forth from a reference form. }\end{array}$ & $\begin{array}{l}\text { drohen, droht '(to) threaten, } \\
\text { (s/he) threatens' }\end{array}$ \\
\hline r_voc & SL & $\begin{array}{l}\text { Vocalic r: When it occurs after a vowel in stressed syllables, } \\
<r>\text { is pronounced [6]. In reduced syllables, }<r>\text { frequently } \\
\text { co-occurs with }<\text { e }>\text { in }<\text { er }>\text {, which is pronounced }[6] .\end{array}$ & dort 'there', Winter 'winter' \\
\hline devoice_final & MO & $\begin{array}{l}\text { Final devoicing: Word forms that are pronounced with fi- } \\
\text { nal devoicing are not spelled phonographically but with the } \\
\text { grapheme for the voiced consonant to signal the morpho- } \\
\text { logical relation between the stem and multisyllabic inflected } \\
\text { forms. }\end{array}$ & Hund, Hunde 'dog, dogs' \\
\hline g_spirant & MO & $\begin{array}{l}\text { g_spirantization: A special case of final devoicing is spi- } \\
\text { rantization of final /g/ to /ç/ and /x/, respectively. Following } \\
\text { Eisenberg (2006)'s overview, it is obligatory after /I/, but not } \\
\text { after /a/. There, /g/ may alternatively be pronounced /k/. }\end{array}$ & winzig 'tiny', Tag 'day’ \\
\hline morph_bound & MO & $\begin{array}{l}\text { Morpheme boundaries: Morphologically complex words } \\
\text { that include the same consonant at the end of one morpheme } \\
\text { and at the beginning of the next include a double consonant } \\
\text { spelling, with one of the consonants pertaining to the first and } \\
\text { the other to the second morpheme, even though articulatorily } \\
\text { speakers typically produce only one phoneme. }\end{array}$ & annehmen 'take on' \\
\hline
\end{tabular}

Table 6: List of key orthographic features (KOF), along with the spelling principles (Princ) they relate to as well as a description and relevant graphemes or examples. The spelling principles are: PG: nondefault phonographic mappings; SL: syllabic principles; MO: morpheme constancy. 


\begin{tabular}{|c|c|c|}
\hline POS & Explanation & Examples \\
\hline ADJA & attributive adjective & $\begin{array}{l}\text { das kaputte Fenster ('the broken window'); } \\
\text { ein süßer Hund ('a cute dog') }\end{array}$ \\
\hline ADJD & adverbial or predicative adjective & $\begin{array}{l}\text { Dodo kommt schnell ('Dodo arrives } \\
\text { quickly'); Er war schnell ('He was quick') }\end{array}$ \\
\hline $\mathrm{ADV}$ & adverb & $\begin{array}{l}\text { schon ('already'); bald ('soon'); doch ('how- } \\
\text { ever'/'yet') }\end{array}$ \\
\hline APPR & preposition; circumposition (left) & $\begin{array}{l}\text { auf dem Bürgersteig ('on the sidewalk'); } \\
\text { ohne Lars ('without Lars') }\end{array}$ \\
\hline APPRART & preposition with an article & $\begin{array}{l}\text { am Ende ('at_the end'); im Teich ('in_the } \\
\text { pond') }\end{array}$ \\
\hline APPO & postposition & ein Jahr lang ('for a year') \\
\hline APZR & circumposition (right) & [no instances in the Litkey Corpus] \\
\hline ART & definite and indefinite article & der/die/das ('the'); ein/eine ('a'/'an') \\
\hline CARD & cardinal number & 16; drei ('three') \\
\hline FM & foreign material & the; happy \\
\hline ITJ & interjection & $\mathrm{hm} ; \mathrm{oh}$ \\
\hline KOUI & $\begin{array}{l}\text { subordinating conjunction with "zu" } \\
\text { and infinitive }\end{array}$ & $\begin{array}{l}\text { um alles zu notieren ('in order to note every- } \\
\text { thing'); anstatt zu ('instead of') }\end{array}$ \\
\hline KOUS & $\begin{array}{l}\text { subordinating conjunction with a sen- } \\
\text { tence }\end{array}$ & weil ('because'); ob ('if'); damit ('so') \\
\hline $\mathrm{KON}$ & coordinating conjunction & und ('and'); oder ('or'); aber ('but') \\
\hline KOKOM & comparative conjunction & als ('than') \\
\hline NN & noun & Hund ('dog'); Freund ('friend') \\
\hline NE & proper name & Lea; Schiller \\
\hline PDS & substituting demonstrative pronoun & Ist das dieser hier? ('Is it this one here?') \\
\hline PDAT & attributive demonstrative pronoun & $\begin{array}{l}\text { in diesem Moment ('in this moment'); dieser } \\
\text { Hund ('this dog') }\end{array}$ \\
\hline PIS & substituting indefinite pronoun & jemand ('someone'); keiner ('nobody') \\
\hline PIAT & $\begin{array}{l}\text { attributive indefinite pronoun without } \\
\text { determiner }\end{array}$ & $\begin{array}{l}\text { kein Anruf ('no call'); irgendein Tier ('some } \\
\text { animal') }\end{array}$ \\
\hline PIDAT & $\begin{array}{l}\text { attributive indefinite pronoun with de- } \\
\text { terminer }\end{array}$ & $\begin{array}{l}\text { die anderen Kinder ('the other kids'); ein } \\
\text { paar Tage ('a few days') }\end{array}$ \\
\hline PPER & irreflexive personal pronoun & ich ('I'); er ('he'); ihm ('him'); mich ('me') \\
\hline PPOSS & substituting possessive pronoun & meins ('mine'); deiner ('yours') \\
\hline PPOSAT & attributive possessive pronoun & $\begin{array}{l}\text { meine Mutter ('my mother'); dein Hund } \\
\text { ('your dog') }\end{array}$ \\
\hline PRELS & substituting relative pronoun & $\begin{array}{l}\text { das Eis; das ('the ice that'); der Mann; der } \\
\text { ('the man who') }\end{array}$ \\
\hline PRELAT & attributive relative pronoun & [no instances in the Litkey Corpus] \\
\hline PRF & reflexive personal pronoun & $\begin{array}{l}\text { sich ('oneself'); einander ('each other'); dich } \\
\text { ('you'); mir ('me') }\end{array}$ \\
\hline
\end{tabular}




\begin{tabular}{|c|c|c|}
\hline PWS & substituting interrogative pronoun & was ('what'); wer ('who') \\
\hline PWAT & attributive interrogative pronoun & $\begin{array}{l}\text { welche Nummer ('which number'); auf } \\
\text { welcher Straße ('on which street') }\end{array}$ \\
\hline PWAV & $\begin{array}{l}\text { adverbial interrogative or relative pro- } \\
\text { noun }\end{array}$ & $\begin{array}{l}\text { warum ('why'); wo ('where'); wann } \\
\text { ('when') }\end{array}$ \\
\hline PAV & pronominal adverb & $\begin{array}{l}\text { dafür ('for that'); dabei ('thereby'); deswe- } \\
\text { gen ('therefore'); trotzdem ('nevertheless') }\end{array}$ \\
\hline PTKZU & "zu" before infinitve & zu rollen ('to roll'); zu sehen ('to see') \\
\hline PTKNEG & particle of negation & nicht ('not') \\
\hline PTKVZ & separated verb-addition & $\begin{array}{l}\text { Lars ruft an ('Lars calls'); Sie hängt Bilder } \\
\text { auf ('She hangs up pictures') }\end{array}$ \\
\hline PTKANT & particle of response & $\begin{array}{l}\text { ja ('yes'); nein ('no'); danke ('thanks'); bitte } \\
\text { ('please') }\end{array}$ \\
\hline PTKA & $\begin{array}{l}\text { particle belonging to adjectives or ad- } \\
\text { verbs }\end{array}$ & zu schnell ('too fast') \\
\hline TRUNC & first part of a composition & [no instances in the Litkey Corpus] \\
\hline VVFIN & finite verb; full & $\begin{array}{l}\text { Lars ruft ('Lars shouts'); Dodo bellte ('Dodo } \\
\text { barked'); }\end{array}$ \\
\hline VVIMP & imperative; full & Guck! ('Look!’); Gib! (‘Give!’) \\
\hline VVINF & infinitive; full & passieren ('(to) happen'); kaufen ('(to) buy') \\
\hline VVIZU & infinitive with "zu"; full & $\begin{array}{l}\text { aufzureißen ('to rip open'); auszuleeren ('to } \\
\text { empty out') }\end{array}$ \\
\hline VVPP & perfect participle & geschrieben ('written'); gefunden ('found') \\
\hline VAFIN & finite verb; auxiliary & du bist ('you are'); Lars hat ('Lars has') \\
\hline VAIMP & imperative; auxiliary & sei leise! ('be quiet!') \\
\hline VAINF & infinitve; auxiliary & $\begin{array}{l}\text { wo er sein könnte ('where he could be'); weil } \\
\text { er die Knochen haben will ('because he wants } \\
\text { to have the bones') }\end{array}$ \\
\hline VAPP & perfect participle; auxiliary & $\begin{array}{l}\text { Dodo ist aggressiv geworden ('Dodo has } \\
\text { become aggressive'); da hat Dodo was zu } \\
\text { Fressen gehabt ('then Dodo has had some- } \\
\text { thing to eat') }\end{array}$ \\
\hline VMFIN & finite verb; modal & $\begin{array}{l}\text { wer darf Dodo mit in die Schule nehmen } \\
\text { ('who is allowed to take Dodo to school'); sie } \\
\text { wollte gerade gehen ('she wanted to go right } \\
\text { now') }\end{array}$ \\
\hline VMINF & infinitive; modal & wollen ('want (to)') \\
\hline VMPP & perfect participle; modal & [no instances in the Litkey Corpus] \\
\hline $\mathrm{XY}$ & non-word; including special symbols & $\begin{array}{l}\text { C. Ronaldo; Hr. ('Mr.'); aules** [unreadable } \\
\text { fragment] }\end{array}$ \\
\hline \$, & comma & 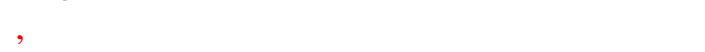 \\
\hline$\$$. & punctuation at the end of a sentence & . ? !! ; : \\
\hline$\$($ & other punctuation; sentence-internal & $"()$ \\
\hline
\end{tabular}

Table 7: STTS tagset (Schiller et al., 1999) used for POS tagging. Examples are taken from the Litkey Corpus. The word in question is marked in red. 